\title{
DIAGNÓSTICO SISTÉMICO Y PROPUESTAS ESTRATÉGICAS PARA EL MEJORAMIENTO COMPETITIVO DEL SECTOR TEXTIL - CONFECCIONES EN LA PROVINCIA DE HUANCAYO
}

\section{SISTEMATIC DIAGNOSIS AND STRATEGIC PROPOSALS TO INCREASE THE COMPETITIVE ADVANTAGE OF THE TEXTILE INDUSTRY AND CONFECTIONS IN THE PROVINCE OF HUANCAYO}

\author{
Miguel Inga Ávila ${ }^{25}$; José Luis Olivera Meza y José Luis Inga Ávila
}

Facultad de Ingeniería de Sistemas

\section{RESUMEN}

El presente trabajo tuvo como finalidad realizar una evaluación de la situación actual del sector industrial productivo textil - confecciones en la provincia de Huancayo. En la investigación se define la identidad sectorial, análisis interno y externo del sector y diamante competitivo de Michael Porter, y posterior a ella se esbozan las respectivas propuestas estratégicas, las mismas que buscan elevar los niveles de competitividad interna y externa. Como herramientas metodológicas se han utilizado, el análisis FODA, lo que ha permitido la definición de las fortalezas y debilidades como parte del análisis interno, y las oportunidades y amenazas como parte del diagnóstico externo. Asimismo, se ha utilizado el diamante competitivo de Michael Porter, para conocer el comportamiento y dinámica sectorial textil tanto actual como futuro. De la misma forma se definió acciones estratégicas de naturaleza técnico - productivo y humana, así como políticas 0 acciones debe desarrollar el gobierno central, regional y local para el logro de la competitividad del sector textil - confecciones de la Provincia de Huancayo. Las herramientas utilizadas permiten tener una idea clara de la situación actual y posteriormente sobre esa base diseñar políticas de desarrollo integral. Las propuestas que se vayan a formular están orientadas a mejorar la posición relativa en el mercado interno y externo del sector textil.

Palabras clave: identidad, competitividad, diamante competitivo, análisis FODA, sector textil

\section{ABSTRACT}

The present work had as purpose to evaluate the current situation of Textil and Confection industry in Huancayo. This research defined the industry identity, Internal and External Analysis of Textile Sector and Michael Porter's Competitive Diamond, and then outlined the respective Strategic Offers, the same ones that seek to increase the levels of internal and external competitiveness. The methodological tools used are FODA Analysis to define Strengths and Weaknesses as part of the internal analysis, and the Opportunities and Threats as part of the external diagnosis. Likewise, Michael Porter's Competitive Diamond was also used, which allowed to determine the behavior and textile industry dynamics. Strategic actions whether they are technically-productive or human were analyzed, as well as policies or actions that must be developed by the central, regional and local government to achieve the desired competitiveness of the textile and confections industry in Huancayo. The used tools allow to develop a clear idea of the current situation so that then, based on findings design the policies of integral development. The proposals that are going to be formulated will be directed towards improving the relative positioning in the internal and external markets of the textile sector.

Key words: Identity, competitiveness, Porter's Competitive Diamond, FODA Analysis, textile industry.

\footnotetext{
25 miguel_inga@hotmail.com
} 


\section{INTRODUCCIÓN}

En las actuales circunstancias de competencia creciente y cambio constante del entorno económico, de mercado y social; las empresas - sin importar su sector ni tamaño consideran un imperativo la necesidad de contar con documentos que guíen y regulen su comportamiento actual y futuro.

Es en este sentido, que los investigadores preocupados en fortalecer las capacidades competitivas, decidieron realizar un diagnóstico situacional que permita identificar las capacidades, limitaciones, oportunidades y riesgos de un conjunto de sectores productivos de toda la provincia en el sector textil.

Para tal efecto, se llevó a cabo reuniones de trabajo con diversos actores del sector textil de la provincia de Huancayo

La metodología de intervención consistió en la realización de mesas de trabajo conformados por empresarios, representantes sectoriales, profesionales, académicos, estudiantes e interesados, reunidos por cada uno de los sectores mencionados. Las herramientas utilizadas fueron el Análisis TASCOI para la definición de la Identidad Sectorial que viene a ser la clarificación de las actividades que actualmente vienen realizando, su propósito como empresarios y como sector; así como el Análisis FODA para la evaluación interna y externa.

Este análisis FODA permite obtener las fortalezas, oportunidades, debilidades y amenazas para cada uno de los sectores estudiados. Del mismo modo se realizó el análisis sectorial mediante el diamante competitivo de Porter, el que busca ilustrar de modo sistémico el comportamiento y dinámica de determinado sector a través de la conjunción de variables como los factores, las industrias relacionadas, la demanda y el propio empresariado.

Posterior a todos estos análisis, se desarrolló el trabajo de gabinete, que permitió esbozar diversas propuestas estratégicas, las mismas que servirán de base para la actualización de Planes de Exportación de naturaleza gubernamental. Los criterios utilizados para estos estudios fueron: análisis de demanda, oferta, potencial exportador, generación de empleo y articulación comercial. El objetivo de este trabajo es realizar una evaluación diagnóstica que permita establecer acciones de carácter estratégico hacia el desarrollo de la competitividad del sector textil - confecciones de la Provincia de Huancayo.

En consecuencia, el presente diagnóstico y propuestas estratégicas plantea a toda la comunidad sectorial, la realización de un trabajo conjunto, articulado y consistente para asumir el reto de potenciar las exportaciones peruanas de los sectores estudiados hacia los principales mercados del mundo, los mismos que dibujen un nuevo escenario al 2015.

Para ello lo fundamental es asumir un compromiso de trabajo conjunto entre toda la comunidad empresarial de la región central de nuestro país, así como un compromiso de apoyo decidido al sector por parte del gobierno y el sector privado, para fortalecer la competitividad sectorial.

\section{DEFINICIÓN DE LA IDENTIDAD SECTORIAL TEXTIL}

Somos empresarios que transformamos fibra de alpaca, oveja y algodón en productos textiles y prendas de vestir para satisfacer gustos y preferencias de los clientes (moda, creación, diseño, innovación, originalidad, combinación, elegancia, distinción) a nivel nacional e internacional, con la finalidad de mejorar la calidad de nuestra familia e intensificar la mano de obra especializada en la comunidad y lograr el posicionamiento en el mercado nacional e internacional llevando un buen control de calidad y servicio con mayor valor agregado de acuerdo a los estándares de orden mundial. 


ANÁLISIS INTERNO
Fortalezas
Capacidad de inversión y reinversión en el sector
-mayoritariamente
Experiencia empresarial en el sector textil
Alto nivel de emprendimientos
Empresas legalmente constituidas.
Los modelos asociativos y consorciales se vienen
llevando con relativo éxito
Experiencia exportadora por parte de algunos
miembros del sector.
Empresarios con mentalidad creativa
Parque de maquinarias antiguas pero operativas
Tecnología de punta para producción industrial
Tecnología de acabados: crochet y palitos
Uso de materias primas $100 \%$ naturales o en
combinaciones según necesidades.
Prendas consideradas productos orgánicos
Productos con alto valor agregado

\section{Debilidades}

Bajos niveles de rentabilidad por la actividad productiva.

No hay poder de negociación frente a los proveedores

Débil poder de negociación ante los clientes.

Desconocimiento de la calidad de materia prima

Proceso productivo empírico con altos costos de manufacturación

Insuficiencia de personal capacitado

Débil nivel de cultura informática

No se cuenta con tecnología de última generación

Heterogeneidad en la disposición de equipos para la producción

Disparidad en calidad y acabado productivo

No se cuenta con certificaciones medioambientales y/o naturales

\section{ANÁLISIS EXTERNO}

Oportunidades

Existencia de empresas capacitadoras y certificadoras
Tendencia al mundo verde

Disponibilidad de centros de desarrollo tecnológico

Tratados de Libre Comercio con diversos países

(USA, Canadá, UE, etc.)

Estabilidad económica

Competencia mundial

Programa Exporta Fácil

Drawback e incentivo a los exportadores

Programas de promoción a las exportaciones

Nueva Ley de MYPES

Programa de compras estatales

Extensión de la temporada invernal en el hemisferio norte

\section{Amenazas}

Excesivas cargas fiscales y presión tributaria

Débil relación con gremios

Disminución de la demanda interna

Alta obsolescencia tecnológica

Retraso cambiario, menos soles por dólar

Creciente nivel de contrabando

Decrecimiento sectorial al 2010

Incremento de la competencia mundial

Extrema dependencia de materia prima

Inelegibilidad en la banca privada a empresas exportadoras

Altas tasas de interés activa

Cambios climáticos

Disconformidad social

\section{DIAMANTE COMPETITIVO DEL SECTOR TEXTIL - CONFECCIONES}

Factores básicos y avanzados: Los factores básicos son auspiciosos pero no son suficientes para el desarrollo del sector, urge la necesidad de potenciar los factores avanzados y demás variables para el logro de la competitividad.

Industrias relacionadas y de apoyo - El cluster: El sector está poco integrado a procedimientos y estructuras relacionadas. Los proveedores no apoyan al dinamismo del sector. Los centros 
de capacitación, investigación y desarrollo se convierten en pilar fundamental para el crecimiento sectorial.

Condiciones de la demanda: La producción actual está orientada a la atención de mercados nacionales, los cuales no se muestran atractivos debido a los bajos niveles de gasto. La demanda exterior se presenta como una alternativa interesante.

Estrategia, estructura y rivalidad de las empresas: La manera cómo se viene desarrollando y manejando el sector está equilibrado. Existen esfuerzos asociativos que permiten generar fuerza y poder de negociación a futuro. Hay que redireccionar la gerencia y el mercado objetivo.

Gobierno: El gobierno va creando oportunidades y promoviendo la actividad sectorial. El Estado es y seguirá siendo un gran comprador (uniformes, ropa escolar, etc.) sin embargo, debe sofisticar sus exigencias, estableciendo altos estándares de calidad y permitiendo la participación de empresas de mayorenvergadura y competitividad. El impulso a la actividad empresarial se dificulta por la corrupción y la burocracia estatal.

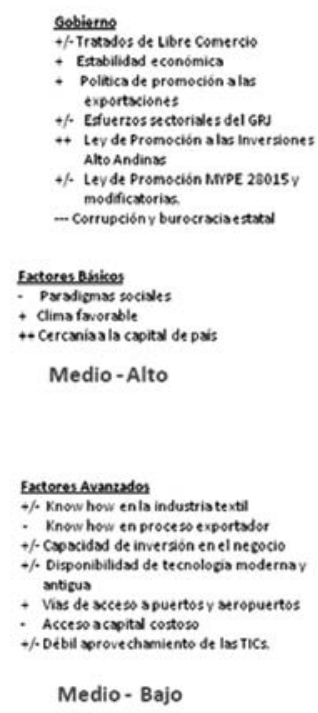

\section{Diamante Competitivo} Sector Textil-Confección

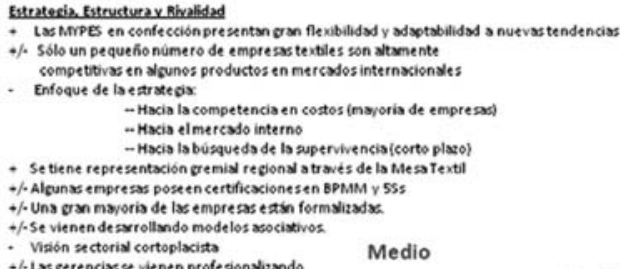

Condiciones de bermanda cenerindo unamariot importaciones ests preciosyun sumento en lacalidad del productolocal

cosconamidoreslocolesen genery no Cosconos Losmercatose teriores sonatess. poder de ne cociación de los clientes locale:

7. capscidad adquiativa de los mercados internacionales. Capsidad adquistiva de los mercastos nstiondes.

- Losclientesprefieren productosorzinicos.

Baja

Arat

\% Disminución tipe de cambio - Nueva crisiseconomica mundial

- Dessotres naturales

- colentamiento doba

- Toma de carreterasy conflictos sociales

\section{PROPUESTAS ESTRATÉGICAS}

Propuestas de desarrollo del sector textil provincia de Huancayo

Implementación de una hilandería moderna y adecuada que permita asegurar el abastecimiento de fibra.

Fortalecimiento de asociaciones y/o consorcios textiles orientados a la mejora del poder de negociación.

Promoción y creación de cadenas productivas que permitan una articulación comercial más fluida.

Realización de convenios con instituciones de apoyo financiero y de inversión para el desarrollo 
empresarial: COFIDE, FOGAPI, etc.

Impulsar programas de capacitación y preparación para obtención de certificaciones (BPMM, 5S, Fair Trade, etc).

Desarrollo de programas de capacitación y fortalecimiento en gestión empresarial, asociatividad y exportación con participación de entidades estatales y privadas como: Promperú, Cámara de Comercio de Huancayo, Sociedad Nacional de Industrias, Gobierno Regional Junín, entre otros.

Programa de capacitación en patronaje, desarrollo de productos, tendencias y diseño de modas.

Realización de ferias y/o desfiles de moda de trascendencia nacional e internacional con la participación de todos los miembros del sector.

Desarrollo de procesos de acercamiento y mejoramiento del contacto comercial con el cliente (participación en ferias, misiones comerciales, pasantías, etc)
Establecimiento de plan de capacitación en tecnologías de la información y comunicaciones conducente a la integración al mundo digital (páginas web, e-mail, etc).

Establecimiento de programas de inteligencia de mercado y análisis de competencia nacional e internacional como base del Benchmarking Competitivo Textil.

Incorporación de tecnologías CAD y CAM a los procesos productivos.

Convenio con instituciones de naturaleza agrícola e industrial para la provisión de tintes naturales.

Inscripción ante OSCE para ser proveedor de bienes al Estado.

Participación organizada en programas de adquisiciones o compras estatales los que permitirán un aseguramiento de las compras.

Diamante competitivo futuro del sector al 2015

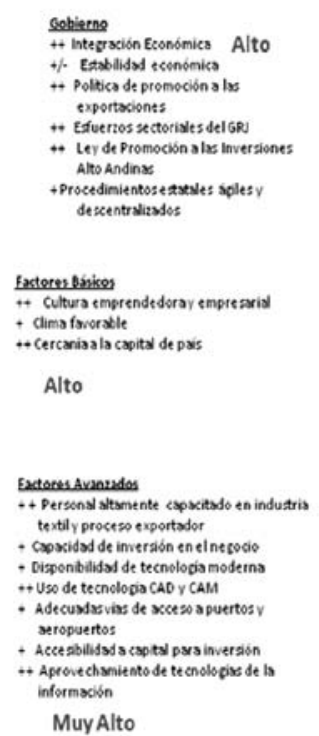

Diamante Competitivo Futuro al 2015 Sector Textil-Confección

Estrateria, Estruitura y Ringlidad

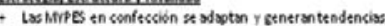

- Creciente numero de enpresas textlesson atrmente sompetitivas tn mercados internxionses de atta exigencia

-. Enfoque de laestrate gix

- Hacia la competencia en dferenciación

Muy Alto

Hacia el mercado enterno, an descuids el interno

- Hacia la búzque da del crecimiento e internscionslitscion flygo plasol

+ Se tiene representación y paticipacion en las politicas de de sarrollo re pional atraves de la

Meastextil y densis cremios

- Lamaroria de enpresas postencertficaionesen BPLAth y 55

Todas las empresas etin fornalizalas

Losmodelos zecistivos son replicados en todx las zons productivas textiles confecciones

- Visón sectoria de larco plaxo

+ Laserenciss se levan de un modo profesional y expecisitzaso

Muy aito

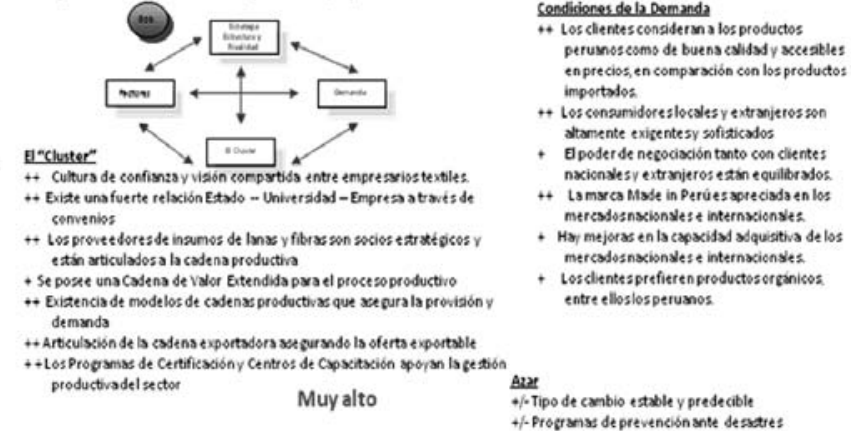


Factores básicos y avanzados: Los factores básicos son auspiciosos y suficientes para el desarrollo del sector, se dispone de lana de ovino y camélidos sudamericanos, tintes y otros insumos para la producción de prendas. Se ha desarrollado los factores avanzados como el conocimiento -a través de la capacitación-, la infraestructura de comunicación -terrestre y aérea-, se ha dinamizado la economía a través de la presencia de diversos bancos y financieras, entre otros que coadyuvan al logro de la competitividad.

Industrias relacionadas y de apoyo - El cluster: El sector está integrado a procedimientos y estructuras relacionadas. Los proveedores de insumos (lanas, sintéticos, mano de obra, etc) y demás actores apoyan al dinamismo del sector. Los centros de capacitación, investigación y desarrollo se convierten en pilar fundamental para el crecimiento sectorial.

Condiciones de la demanda: La producción actual está orientada a la atención de mercados nacionales e internacionales. Al mismo tiempo, los niveles de ingresos familiares de los mercados nacionales han mejorado e incrementan la demanda.

Estrategia, estructura y rivalidad de las empresas: Existen esfuerzos asociativos formales que permiten generar fuerza y poder de negociación. La competencia ha pasado de ser interna a externa debido a la presencia de los consorcios empresariales en mercados internacionales.

Gobierno: El Gobierno va creando oportunidades y promoviendo la actividad sectorial. El Estado es y seguirá siendo un gran comprador (uniformes, ropa escolar, etc.) sin embargo, debe sofisticar sus exigencias, estableciendo altos estándares de calidad y permitiendo la participación de empresas de mayor envergadura y competitividad. Los niveles de corrupción y burocracia estatal han disminuido.
La exportación se convierte en una alternativa viable y sostenible para el desarrollo de los diversos sectores productivos y de servicios de la Región Junín.

Los sectores con mejores posibilidades de seguir avanzando y creciendo en los procesos de exportación son: textiles y confecciones, café -con incidencia en cafés especiales-, trucha, cacao, artesanía y flores; razón por la cual merecen realizarse los respectivos Planes Operativos de Producto.

La obtención de la Identidad Sectorial mediante la técnica TASCOI, permite ampliar los criterios bajo los cuales se vienen llevando adelante los negocios. Con esta técnica, los empresarios no sólo han definido a qué se dedican, sino también qué venden, en qué contexto, qué productos, qué valor agregado brindan, qué necesidades satisfacen, etc; permitiendo una visión mucho más amplia de sus respectivas unidades productivas.

Se evidencia en todos los sectores analizados, una gestión empírica de las empresas, sin visión de largo plazo, poco espíritu de asociatividad y sobre todo una extrema dependencia de las ayudas estataleso de organismos internacionales.

En virtud a lo anterior, la asociatividad se convierte en el mecanismo de fortalecimiento de la actividad empresarial con miras a la exportación.

La participación de los diferentes entes involucrados en el quehacer empresaria tales como: Gobierno regional, Gobiernos locales, Promperú, Sociedad Nacional de Industrias, Cámaras de Comercio, Asociación de Exportadores, gremios empresariales, universidades, ONGs, etc. son vitales para la generación de una masa crítica de exportadores, capaces de atender mercados internacionales y generar empleo y desarrollo sostenible. La labor del Gobierno no debe ser proteccionista, sino más bien de promotor de la inversión y delineador de las condiciones favorables de mercado. 


\section{REFERENCIAS BIBLIOGRÁFICAS}

Ackoff, Russell. Planificación de la empresa del futuro. Ed. Limusa.- Noriega Ed, México. 1997.

Espejo, Raúl y otros. Viable System Model: Interpretations and Applications of Stafford Beer's. $1^{\circ}$ Ed. John Wiley \& Sons Inc. 1989

Indacochea, Alejandro y otros. Junín Competitivo: Porter, Michael E. Ventaja competitiva de las Valle del Mantaro. $1^{\circ}$ Edición. Centrum Católica. naciones. Editorial Vergara. 1991 2006

Indacochea, Alejandro y otros. Junín Competitivo: Valle del Mantaro. $1^{\circ}$ Edición. Centrum Católica. 2006

Inga Avila, Miguel Fernando y Olivera Meza, José Luis. Plan de Desarrollo Empresarial en las mypes de Huancayo. Trabajo de Investigación. Universidad Nacional del Centro del Perú. 1998

Monitor Company. Diamante competitivo del sector textil-Confección Colombiano. 1997

Monitor Company. Diamante competitivo del sector textil-Confección Venezolano. 1996

Porter, Michael E. Ventaja competitiva. $1^{\circ}$ Ed. Editorial Deusto. 2006

Porter, Michael E. Ser competitivo. $1^{\circ}$ Ed. Editorial Bilbao. 1999 